\title{
Composição e dinâmica de grupos de Alouatta guariba clamitans Cabrera (Primates, Atelidae) em Floresta Ombrófila Mista no Estado do Paraná, Brasil ${ }^{1}$
}

\author{
João M. D. Miranda ${ }^{2} \&$ Fernando C. Passos ${ }^{2}$ \\ ${ }^{1}$ Contribuição número 1542 do Departamento de Zoologia, Universidade Federal do Paraná. \\ ${ }^{2}$ Laboratório de Biodiversidade, Ecologia e Manejo de Animais Silvestres, Departamento de Zoologia, Universidade Federal do \\ Paraná. Caixa Postal 19020,81531-980 Curitiba, Paraná, Brasil.E-mail: guaribajoao@yahoo.com.br, fpassos@ufpr.br
}

\begin{abstract}
Composition and dynamics of Alouatta guariba clamitans Cabrera, groups (Primates, Atelidae) on an Araucaria Pine Forest remnant on Paraná State, Brazil. Species in the genus Alouatta Lacépède, 1799 are social, and their groups display considerable variation in their age and sex composition. Little is known about the social organization of groups of Alouatta guariba clamitans Cabrera, 1940. This study was carried out between February 2002 and June 2004 in the Chácara Payquerê, located in the municipality of Balsa Nova in the state of Paraná (Southern Brazil). The vegetation in this area is the Araucaria Pine Forest, and this reserve is located within the Area for Environmental Protection of the Devonian Scarp. During observations, groups were identified and their sex/age composition and dynamics were recorded. Six groups were observed over the course of three years. Average composition values were 6.33 individuals/group; 1.47 adult male (AM)/group; 0.46 subadult male/group; 2.2 adult females (AF)/group; 1.13 juvenile II/group; 0.8 juvenile I/group, and 0.27 infant/ group. In order to describe the sex/age composition of this species, the data obtained in the present study were combined with information on 66 additional groups of $A$. g. clamitans available in the literature, totaling 72 groups. Ensemble values obtained for this species are 6.25 individuals/group; $1.39 \mathrm{AM} /$ group; $2.26 \mathrm{AF} /$ group and 2.62 immature individuals/group. With respect to the dynamics of the groups, the following data were recorded: 11 births, 25 age class changes, 4 solitary individuals, 1 death/disappearance, and the fission of one group into two. Therefore, the current study described the the sex/age composition of $A$. g. clamitans groups, as well as some aspects of their dynamics.
\end{abstract}

KEY WORDS. Brown-howler monkey, ecology of populations.

RESUMO. As espécies do gênero Alouatta Lacépède, 1799 são sociais e formam grupos que mostram grandes variações na sua composição sexo-etária. Alouatta guariba clamitans Cabrera, 1940 é uma das espécies com escassos trabalhos que mostrem aspectos sociais deste primata. $O$ estudo foi conduzido na Chácara Payquerê, localizada no Município de Balsa Nova, Estado do Paraná, Sul do Brasil.A área é inserida no contexto da Floresta Ombrófila Mista e faz parte da Área de Proteção Ambiental da Escarpa Devoniana. O trabalho foi conduzido de fevereiro de 2002 até junho de 2004, identificando grupos, registrando a composição sexo-etária e sua dinâmica. Foram identificados seis grupos na área de estudo, no decorrer dos três anos as médias de suas composições foram: 6,33 indivíduo/grupo; 1,47 Macho Adulto (MA)/grupo; 0,46 Machos subadultos/grupo; 2,2 Fêmeas Adultas(FA)/ grupo; 1,13 Juvenis II/grupo; 0,8 Juvenis I/grupo e 0,27 Infantes/grupo. Para uma análise sobre a composição sexo-etária da espécie, os dados obtidos neste trabalho foram somados a 66 outros grupos de A. g. clamitans disponíveis na literatura (totalizando 72 grupos), sendo que as médias para a espécie foram: 6,25 indiv./grupo; 1,39 MA/grupo; 2,26 FA/grupo e 2,62 Indivíduos Imaturos/grupo. Quanto à dinâmica dos grupos registraramse: 11 nascimentos; 25 mudanças de categoria etária; 4 indivíduos transeuntes; 1 morte/desaparecimento e a quebra de um grupo em dois. Este trabalho analisa a composição sexo-etária de $A$. g. clamitans além de discutir alguns aspectos da dinâmica dos grupos.

PALAVRAS CHAVE. Bugio-ruivo, ecologia populacional.

Os representantes do Gênero Alouatta Lacépède, 1799 figuram entre os maiores primatas neotropicais, com seu peso variando entre 4 e $7 \mathrm{~kg}$. Os machos são maiores que as fêmeas e apre- sentam uma pelagem mais densa, principalmente na região gular, onde se forma uma espessa barba. Na sub-espécie Alouatta guariba clamitans Cabrera, 1940, além do tamanho, nota-se um 
dicromatismo sexual na coloração da pelagem, onde os machos adultos apresentam-se ruivos, vermelhos ou alaranjados e as fêmeas adultas e os indivíduos imaturos são geralmente castanhos escuros (von Ihering 1914, Cabrera \& Yepes 1960).

As espécies do gênero Alouatta são sociais e formam grupos que podem variar muito entre as espécies e menos intraespecificamente. Os números gerais de indivíduos por grupo são: Alouatta palliata (Gray, 1849) 5-45; A. pigra Lawrence, 1933 2-17; A. belzebul (Linnaeus, 1766) 2-8; A. caraya (Humboldt, 1812) 3-19; A. seniculus (Linnaeus, 1766) 9-26 e A. guariba (Humboldt, 1812) 2-11 (Neville et al. 1988, Emmons \& Feer 1997, TREves 2001). Essa variação pode ser ligada à ampla distribuição do gênero (a maior dentre os primatas neotropicais), que vai do México ao Sul do Brasil e Norte da Argentina, ocupando diversos ambientes, o que pode exigir estratégias comportamentais diferentes (von Ihering 1914, Cabrera 1957, Mendes 1989, AuricChio 1995, EMmons \& FeER 1997).

A estrutura sexo-etária dos grupos de primatas é ligada ao padrão de dispersão de machos e de fêmeas. Para Alouatta sabe-se que ambos os sexos migram, podendo formar novos grupos ou simplesmente integrar-se a um outro grupo (BRоскетT et al. 2000, Ostro et al. 2001).

Normalmente, os grupos de Alouatta são compostos por mais fêmeas que machos adultos, podendo ser formados por: pares heterossexuais, um macho e várias fêmeas ("unimale") ou vários machos e várias fêmeas ("multimale"). Neste último caso é aceito que os machos apresentem uma gradação de idade, ou seja, um macho é o dominante ou alfa do grupo, e os outros machos devem ter parentesco com o macho alfa e são mais novos que esse (AltMAnN 1959, Neville et al. 1988, Rudran \& Fernandez-Duque 2003, Wang \& Milton 2003).

Alouatta é o gênero mais bem estudado entre os primatas neotropicais (Neville et al. 1988). Muito embora, A. g. clamitans estar entre as mais bem conhecidas do gênero (BICCA-MARQues 2003), são poucos os estudos de longa duração que forneçam dados sobre as dinâmicas sociais de muitas espécies de primatas do Novo Mundo. Somente trabalhos com A. palliata, A. seniculus e Brachyteles hypoxanthus (Kuhl, 1820) apresentam tal acompanhamento (Strier et al. 1993, Rudran \& Fernandez-Duque 2003, DiAs \& STRIER 2003).

O objetivo deste estudo foi registrar as composições sexoetárias dos grupos de bugios presentes em uma área de Floresta Ombrófila Mista, bem como verificar as mudanças nas categorias etárias e observar a dinâmica social dos grupos ao longo do tempo. Foi também objetivo encontrar um padrão de composição social para A. g. clamitans compilando dados de diversos autores, considerando a subespécie em toda sua distribuição geográfica.

\section{MATERIAL E MÉTODOS}

\section{Área de estudo}

O presente estudo foi realizado na Chácara Payquerê: Centro de Educação Ambiental e Apoio à Pesquisa (90ha), lo- calizada no Distrito do Bugre, Município de Balsa Nova $\left(25^{\circ} 29^{\prime} 52^{\prime \prime} \mathrm{S}\right.$ e $\left.49^{\circ} 39^{\prime} 24^{\prime \prime} \mathrm{W}\right)$ a aproximadamente $40 \mathrm{Km}$ de Curitiba, PR (Fig. 1). A área encontra-se inserida em um remanescente de Floresta Ombrófila Mista (700 ha) e faz parte da Área de Proteção Ambiental da Escarpa Devoniana (МıкıсH \& BérNILS 2004), apresentando temperatura média anual de $17,9^{\circ} \mathrm{C}$ e um índice pluviométrico em torno de $1600 \mathrm{~mm}$.

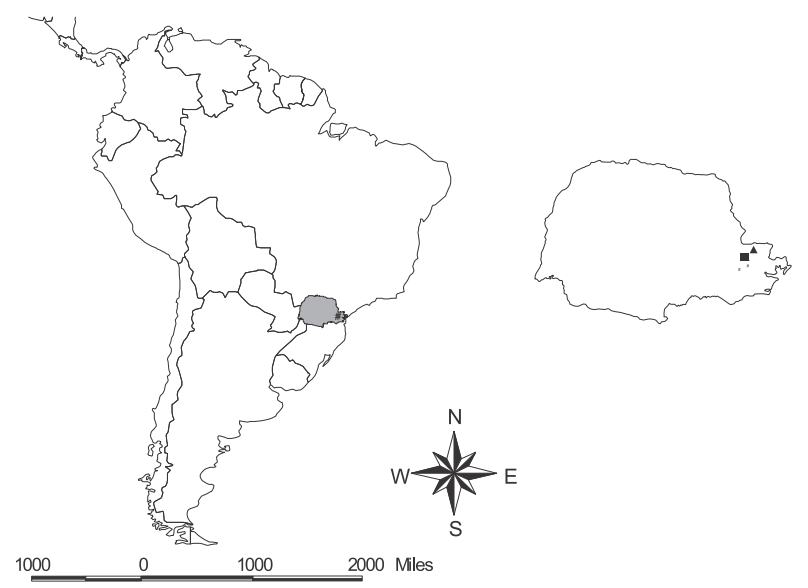

Figura 1. Mapa Localizando a Cidade de Balsa Nova (quadrado) e Curitiba (triângulo), no estado do Paraná, no Brasil e na América do Sul.

\section{Coleta de dados}

O trabalho foi realizado de fevereiro de 2002 a junho de 2004. Foram percorridas todas as principais trilhas, beiras de rios e outras formas de acesso às imediações da Chácara Payquerê, visando conhecer e localizar todos os grupos de A. g. clamitans residentes na área de estudo e adjacências, bem como suas composições sexo-etárias. Os grupos foram definidos com base no local de observação e na composição sexo-etária dos indivíduos, bem como nas repetidas observações de cada grupo. A cada observação foram anotados: a data, o local, o horário inicial e final da observação e a composição sexo-etária do grupo sendo que as classes sexo-etárias foram as mesmas utilizadas por Mendes (1989): Infante (Inf) - indivíduo dependente da mãe, sendo carregado na barriga ou nas costas, todo o tempo ou na maior parte deste; Juvenil I (JI) - indivíduo de tamanho próximo ao infante, raramente carregado pela mãe, somente em casos de estresse; Juvenil II (JII) - indivíduo completamente independente e de tamanho maior que o JI e menor que fêmeas adultas; Macho sub-adulto (MSA) - maior que a fêmea adulta e menor ou igual ao macho, mas com o saco escrotal cobertos por pelagem, de coloração do corpo geralmente menos vermelha que o MA; Fêmea adulta (FA) - coloração escura, variando do preto ao castanho avermelhado, genitália descoberta de forma triangular ou levemente arredondada quando vista de um ângulo ventroposterior. 
Macho adulto (MA). Nitidamente maior que fêmeas adultas, barba longa, pelagem bem densa, cuja coloração que pode variar do vermelho acastanhado, passando pelo ruivo até o alaranjado; e com o saco escrotal bem desenvolvido e à mostra.

Como a composição dos grupos é dinâmica no tempo e no espaço, foi tomada uma amostra pontual da composição ao final dos anos de 2002 e 2003 e em agosto de 2004 para se gerar a tabela de composição dos grupos estudados (Tab. I) e analisar os dados, para seu entendimento. Para as comparações e análise dos dados obtidos neste trabalho e em outros foram utilizadas as composições descritas dos grupos e não as médias das composições.

Todos os animais foram identificados individualmente com base em suas classes sexo-etárias, tamanho corpóreo, colorações diferenciadas e marcas individuais.
No decorrer do período de estudo foram observadas de maneira oportunista (ad libitum, Attmann 1974) e descritas as mudanças etárias, nascimentos, desaparecimentos, quebra (desagregamento) de grupos, bem como confrontos agonísticos intra-grupais e intergrupais.

Dentre o gênero Alouatta, a média do número de indivíduos por grupo varia profundamente entre as espécies e também intraespecificamente (Neville et al. 1988, Emmons \& FeER 1997, Nowak 1999, Treves 2001). Portanto, neste trabalho serão analisados somente os dados de tamanho e composição de grupos de A. g. clamitans. Ao todo foram analisadas as médias de 72 grupos de A. g. clamitans, ao longo da distribuição geográfica da espécie e em diferenciados ambientes, permitindo inferir dados sobre a composição e estrutura sexo-etária padrão da espécie. Para comparar os dados sobre outras categorias sexo-etárias, que não as

Tabela I. Composição de grupos de Alouatta guariba clamitans observados na Chácara Payquerê, Município de Balsa Nova, Paraná, durante três anos de estudo.

\begin{tabular}{|c|c|c|c|c|c|c|c|}
\hline Nome do grupo & $\begin{array}{c}\mathrm{N}^{\circ} \text { de } \\
\text { indivíduos }\end{array}$ & $\begin{array}{l}\text { Machos } \\
\text { adultos }\end{array}$ & $\begin{array}{c}\text { Machos } \\
\text { subadultos }\end{array}$ & $\begin{array}{l}\text { Fêmeas } \\
\text { adultas }\end{array}$ & Juvenis II & Juvenis I & Infantes \\
\hline \multicolumn{8}{|l|}{ Composição dos grupos em 2002} \\
\hline Forninho & 6 & 2 & - & 3 & - & 1 & - \\
\hline Bica & 6 & 1 & 1 & 2 & 1 & 1 & - \\
\hline Cachoeira & 4 & 1 & - & 2 & - & 1 & - \\
\hline Pinheiro & 8 & 2 & 1 & 3 & 1 & - & 1 \\
\hline Estrada & 6 & 1 & 1 & 2 & 1 & 1 & - \\
\hline Indivíduo solitário I & $1\left(^{*}\right)$ & - & - & 1 & - & - & - \\
\hline Indivíduo solitário II & $1\left(^{*}\right)$ & - & - & 1 & - & - & - \\
\hline Média dos grupos & 6 & 1,4 & 0,6 & 2,4 & 0,6 & 0,8 & 0,2 \\
\hline \multicolumn{8}{|l|}{ Composição dos grupos em 2003} \\
\hline Forninho & 10 & 2 & - & 3 & 3 & 1 & 1 \\
\hline Bica & 7 & 2 & - & 2 & 2 & 1 & - \\
\hline Cachoeira & 6 & 1 & - & 2 & 1 & 2 & - \\
\hline Pinheiro & 5 & 2 & - & 2 & - & 1 & - \\
\hline Patropí & 4 & 1 & 1 & 1 & - & 1 & - \\
\hline Indivíduo solitário II & $1\left(^{*}\right)$ & - & - & 1 & - & - & - \\
\hline Indivíduo solitário III & $1\left(^{*}\right)$ & - & 1 & - & - & - & - \\
\hline Média dos grupos & 6,4 & 1,6 & 0,2 & 2 & 1,2 & 1,2 & 0,2 \\
\hline \multicolumn{8}{|l|}{ Composição dos grupos em 2004} \\
\hline Forninho & 10 & 1 & 1 & 3 & 2 & 2 & 1 \\
\hline Bica & 7 & 2 & 1 & 3 & 1 & - & - \\
\hline Cachoeira & 6 & 1 & - & 2 & 3 & - & - \\
\hline Pinheiro & 5 & 2 & - & 2 & 1 & - & - \\
\hline Patropí & 5 & 1 & 1 & 1 & 1 & - & 1 \\
\hline Indivíduo solitário IV & $1\left(^{*}\right)$ & - & - & 1 & - & - & - \\
\hline Média dos grupos & 6,6 & 1,4 & 0,6 & 2,2 & 1,6 & 0,4 & 0,4 \\
\hline $\begin{array}{l}\text { Média e porcentagem dos grupos durante } \\
\text { os três anos }\end{array}$ & 6,3 & $1,5(23 \%)$ & $0,5(7 \%)$ & $2,2(35 \%)$ & $1,1(18 \%)$ & $0,8(13 \%)$ & $0,3(4 \%)$ \\
\hline
\end{tabular}

*) Não participa das médias. 
adultas, tornou-se necessário criar uma categoria geral denominada indivíduos imaturos (Ind. Im.), com o objetivo de padronizar os dados apresentados de diversas formas pelos autores.

\section{RESULTADOS}

\section{Composição dos grupos}

No decorrer do ano de 2002 foram avistados na área de estudo e nas adjacências, cinco grupos de bugios e duas fêmeas solitárias (indivíduo solitário I e II). O tamanho médio dos grupos foi de seis indivíduos por grupo, com uma média de 1,7 FA por MA (Tab. I).

Em 2003 também foram avistados cinco grupos (embora um dos grupos observados em 2002 não tenha mais sido visto na área, houve a fragmentação de um outro grupo em dois), uma fêmea solitária (indivíduo solitário II) avistada novamente, além da presença de um macho sub-adulto (indivíduo solitário III) nas imediações da Chácara. A média de indivíduos por grupo foi 6,4 e houve uma relação de 1,2 FA por MA (Tab. I).

Em 2004, foram observados os mesmos cinco grupos de 2003 e uma terceira fêmea solitária (indivíduo solitário IV). A média de indivíduos por grupo, nesse ano, foi de 6,6, sendo que houve uma relação de 1,6 FA por MA (Tab. I).

A média geral de indivíduos por grupo durante todo o período de estudo foi 6,3 e a porcentagem de machos adultos, fêmeas adultas e indivíduos imaturos (foi, respectivamente, de: $23 \%$ de MA, 35\% de FA e $42 \%$ de indivíduos imaturos (Tab. I).

De forma geral, os grupos de $A$. g. clamitans tiveram a tendência de formar grupos pequenos com média de $6,3 \pm 2,3$ indivíduos, com a moda igual a 6 e variando em tamanho de 2 a 12 indivíduos.

O tamanho médio e a composição dos grupos encontradas neste trabalho mostraram-se congruentes com o observado por outros autores (Silva Jr. 1981, Mendes 1989, Chiarello 1993, GaletTi et al. 1994, Steinmetz \& SouZa 1998, Jardim \& Oliveira 2000, Limeira 2000, Silveira \& Codenotti 2001, Steinmetz 2001, Fortes 2002, Aguiar et al. 2003) (Tab. II).

\section{Dinâmica dos grupos}

Grupo Forninho. Durante o início dos estudos (fevereiro de 2002), o Grupo Forninho contava com seis indivíduos: um MA, um MSA, três FA's e um JI. Em 2003, foram registrados quatro nascimentos; dois em janeiro, um em julho e outro em setembro. Em 2004 desapareceu o macho adulto 1 (alfa) deste grupo, provavelmente alvo de caça ilegal (conforme informações de moradores locais). Ainda em 2004 ocorreu um novo nascimento. As demais modificações foram apenas mudanças de classe etária: uma em 2002, e três em 2003 e quatro em 2004 (Tab. I e Fig. 2).

Grupo Bica. Em 2002 o grupo apresentou seis indivíduos: um MA, um MSA, duas FA's, um JII e um JI. Em 2003 ocorreu um único nascimento. Houve também mudanças de categorias etárias: uma mudança em 2002, duas em 2003 e três em 2004 (Tab. I e Fig. 3).

Revista Brasileira de Zoologia 22 (1): 99-106, março 2005
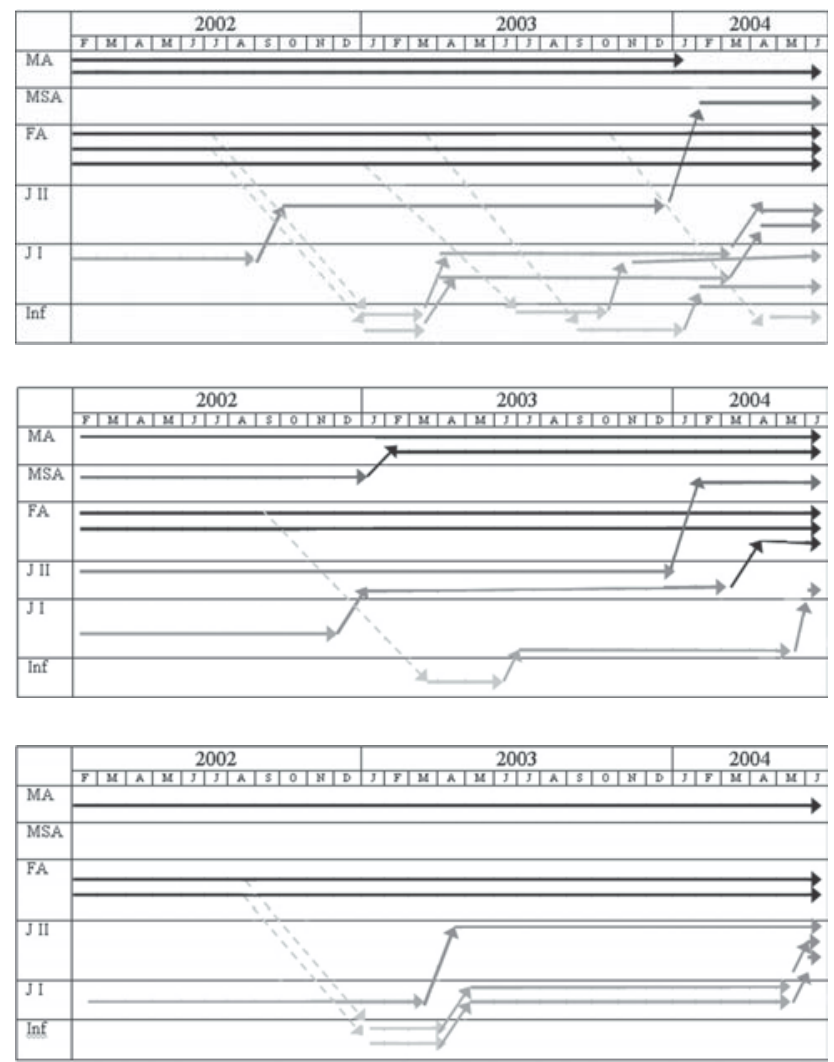

Figuras 2-4. Esquema da dinâmica do Grupo (2) Forninho, (3) Bica e (4) Cachoeira, no decorrer dos dois anos e meio do estudo. Setas tracejadas indicam da gravidez ao nascimento.

Grupo Cachoeira. Em 2002 este grupo era composto por quatro indivíduos: um MA, duas FA's e um JI. Em 2003 registrou-se dois nascimentos. Ocorreram também, três mudanças de categoria etária em 2003 e duas em 2004 (Tab. I e Fig. 4).

Grupo Pinheiro. Este grupo, em 2002, constituía-se de oito indivíduos: dois MA's, um MSA, três FA's, um JII e um Inf. Registrou-se um nascimento em 2002 e outro em 2003. Em junho de 2003 deu-se a passagem do macho de categoria subadulto para a categoria adulto, que por sua vez deu início a uma série de confrontos entre machos. Em agosto desse mesmo ano, um dos machos adultos (Patropí) separou-se do grupo, ocorrendo confrontos agonísticos ritualizados (por meio de vocalizações tipo rugido) contra seu antigo grupo e contra outro grupo vizinho (Grupo Bica). Em setembro, uma das FAs do grupo Pinheiro, acompanhada por um JI e um MSA, começou a se agregar durante o dia com o macho isolado (Patropí), porém ao anoitecer, juntava-se novamente ao seu grupo de origem. Já em outubro essa fêmea com seus acompanhantes juntaram-se em definitivo ao macho isolado formando um novo grupo (Grupo Patropí). A partir de outubro de 2003, o Grupo Pinheiro passou a contar com cinco membros, devido à 
separação de quatro de seus componentes e ao nascimento de um infante. Houve, ainda, quatro mudanças de classe etária em 2003 e uma em 2004 (Tab. I e Fig. 5).

Grupo Patropí. Conforme descrito anteriormente, o Grupo Patropí formou-se em definitivo em outubro de 2003, constituindo-se de quatro indivíduos: um MA, um MSA, uma FA e um JI. Em 2004 ocorreu uma mudança de categoria etária e um nascimento, resultando em um grupo composto por cinco indivíduos (Tab. I e Fig. 5).

Grupo Estrada. Este grupo foi visto somente no ano de 2002 e contava com seis indivíduos: um MA, um MSA, duas FA's, um JII e um JI.

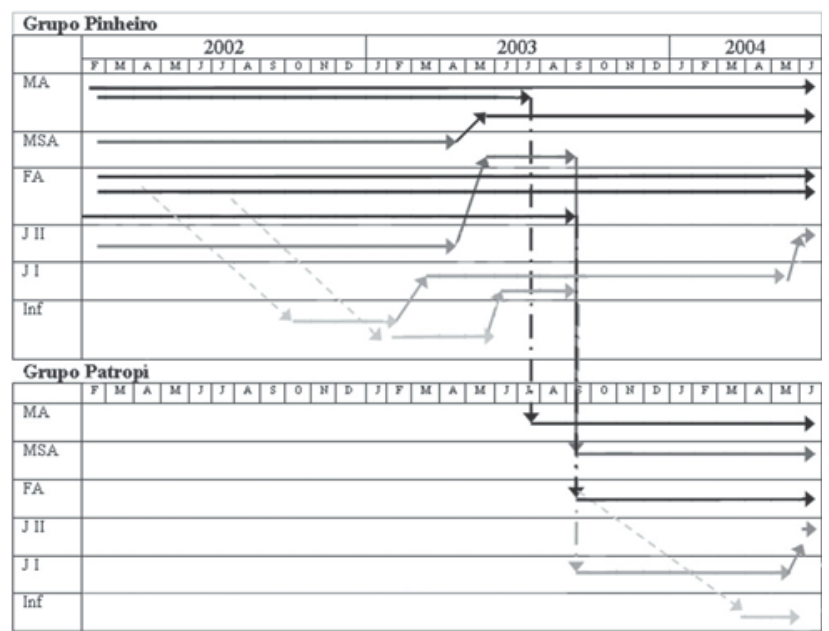

Figura 5. Esquema da dinâmica do Grupo Pinheiro, sua ruptura e formação do Grupo Patropí, no decorrer dos dois anos e meio do estudo. Setas tracejadas indicam da gravidez ao nascimento. Setas com traço e pontos indicam mudança de grupo.

\section{DISCUSSÃO}

\section{Composição de grupos}

Na Chácara Payquerê, assim como nos outros estudos os grupos foram formados por um ou dois MA's (Média de 1,39 \pm 0,2, e moda =1). Em alguns locais (P. E. Cantareira e Chácara Payquerê), aparecem médias de MAs por grupo próximas de 1,5, demonstrando que a formação de grupos com dois MA's talvez seja comum em determinadas populações. Mesmo sendo raros os casos com 3 MAs por grupo, eles ocorreram em três grupos no estudo de SILVA JR. (1981) e no presente estudo. Neste último caso, essa ocorrência foi por um pequeno período de tempo (aproximadamente dois meses), sendo que logo ocorreu a fragmentação do grupo, com a posterior formação de um novo grupo. É possível que este acontecimento foi devido à competição entre os machos, surgindo então um grupo com dois MAs (Grupo Pinheiro) e outro com um só MA (Grupo Patropí).

O número de MAs por grupo mostrou-se positivamente correlacionado ao número de FAs por grupo $(\mathrm{r}=0,36 ; \mathrm{t}=3,26$; $\mathrm{p}<0,05$; g.l. = 70). Assim, talvez quanto maior número de FA's maior a necessidade de os MA's defender o grupo contra a invasão de outros machos. Bonvicino (1989) mostrou a relação entre o número de MA e o tamanho do grupo, além de levantar a hipótese de que em áreas onde ocorre uma alta competição por recursos alimentares pode ser vantajoso ter dois MA's por grupo, como defesa de recurso. A. A. B. MARQues (dados não publicados) também acredita que mais que um MA por grupo esteja relacionado à defesa de recursos e FA's.

Neste trabalho a média de FA por grupo foi 2,2, estando em conformidade com os dados observados na tabela II. Para A. g. clamitans, de forma geral, houve uma tendência para a formação de grupos com duas fêmeas adultas (média $=2,3 \pm$ 1,1 e moda $=2$ ).

No estudo da Chácara Payquerê a média de indivíduos imaturos foi 2,6, divididas em: MAS's, JII's, JI's e Inf's. Os resultados deste trabalho mostraram-se novamente dentro do desvio padrão obtido para os dados dos dez trabalhos aqui analisados. Os indivíduos imaturos apareceram aproximadamente com a mesma média encontrada para o taxa (Tab. II).

\section{Dinâmica dos grupos}

As categorias menos representadas neste estudo foram Infantes e Juvenis I. Talvez isso seja devido ao curto período de tempo em que o animal é alocado dentro destas categorias. O número de indivíduos imaturos foi positivamente correlacionado ao número de FA's por grupo $(\mathrm{r}=0,625 ; \mathrm{t}=2,88 ; \mathrm{p}<0,05$; g.l. =13). Para $A$. g. clamitans a correlação também é válida $(\mathrm{r}=$ 0,392; t = 3,62; p < 0,05; g.l. = 71). Ou seja, um maior número de FA's aumenta a taxa de indivíduos imaturos por grupo. A razão Indivíduos Imaturos/FA foi igual a 1,2, e pode demonstrar a relação entre o número de FA's e a taxa de natalidade. Sendo que a taxa anual (referente a 2003) de natalidade foi de 0,72 nascimentos por FA. Esta taxa de natalidade se mostra semelhante aos encontrados por M. M.A. JARDIM (dados não publicados), 0,75 e 0,45 para 2000 e 2001 respectivamente. Para Alouatta seniculus (Linnaeus, 1758) CRоскетT \& RUdRAN (1987) verificaram uma taxa que variou de 0,49 a 0,91 em vários anos de estudo. Os relativamente altos valores encontrados no presente estudo pode indicar um crescimento populacional.

Não houve uma sazonalidade bem definida para os nascimentos $\left(\chi^{2}=3,17 ; \mathrm{p}>0,05 ;\right.$ g.l. $\left.=3\right)$, ocorrendo em todo o ano $(\mathrm{N}=5$ no verão, $\mathrm{N}=3$ no outono, $\mathrm{N}=2$ no inverno e $\mathrm{N}=$ 1 na primavera).

Em algumas espécies de primatas somente um dos sexos ou classe sexo-etária migra. Em outras espécies ambos os sexos migram, sendo mais comum que estas migrações sejam feitas por indivíduos imaturos (Dunbar 1988). Para Alouatta é sabido que tanto machos como fêmeas migram (Bonvicino 1989, Calegaro-Marques \& Bicca-Marques 1996, Brockett et al. 2000, Ostro et al. 2001, MARQues 2003). Esta migração é ligada à competição intra-específica por recursos (MARQUES 2003). Neste trabalho não foi registrado nenhum indivíduo imigrando para os

Revista Brasileira de Zoologia 22 (1): 99-106, março 2005 
Tabela II. Comparação entre a composição e a estrutura dos grupos de Alouatta guariba clamitans em várias áreas de estudo no Brasil.

\begin{tabular}{|c|c|c|c|c|c|c|}
\hline $\begin{array}{c}\text { Média de } \\
\text { indivíduos/grupo }\end{array}$ & M. A. & F. A. & I. I. & $\begin{array}{c}\text { Número de } \\
\text { grupos estudados }\end{array}$ & Área de estudo & Autor \\
\hline 4,00 & 1,00 & 1,00 & 2,00 & 1 & Sta. Genebra, Campinas, SP & Galemti et al. (1994) \\
\hline 4,00 & 1,00 & 2,00 & 1,00 & 1 & Mata Boa Vista, RJ & LIMEIRA (2000) \\
\hline 4,00 & 1,00 & 1,60 & 1,60 & 2 & Mata Doralice, Ibiporã, PR & AGUiAR et al. (2003) \\
\hline $4,90^{*}$ & $1,00^{*}$ & $2,00^{*}$ & $3,00^{*}$ & $15^{*}$ & Sta. Genebra, Campinas, SP & Chiarello (1993) \\
\hline 5,76 & 1,76 & 2,36 & 1,64 & 25 & P. E. da Cantareira, SP & SILVA JR. (1981) \\
\hline 5,83 & 1,17 & 2,17 & 3,34 & 12 & P. E. Intervales, SP & SteinMetz (2001) \\
\hline 6,00 & 1,00 & 2,00 & 3,00 & 1 & Sta. Genebra, Campinas, SP & Steinmetz \& Souza (1998) \\
\hline 6,00 & 1,00 & 2,00 & 3,00 & 1 & P. E. Itapuã, RS & Silveira \& CodenotTI (2001) \\
\hline 6,33 & 1,47 & 2,20 & 2,66 & 6 & Bugre, Balsa Nova, PR & Este trabalho \\
\hline 6,79 & 1,16 & 2,32 & 3,31 & 19 & R. B. Caratinga, MG & Mendes (1989) \\
\hline 9,00 & 1,50 & 3,00 & 5,00 & 2 & Santa Maria, RS & FORTES (2002) \\
\hline 11,00 & 2,00 & 3,00 & 6,00 & 1 & E. E. Aracurí, RS & JARDIM \& OLIVEIRA (2000) \\
\hline $6,25^{\star *}$ & 1,39 & 2,26 & 2,62 & $86(72)^{\star \star \star}$ & & Médias \\
\hline
\end{tabular}

*) a média de tamanho de grupo apresentada por CHIARELlo (1993) é relativa a 15 grupos encontrados na área do estudo. Mas somente é descrita a composição de um dos grupos, aqui somente este valor será analisado. ${ }^{* *}$ ) as médias foram feitas com base em todos os grupos apresentados pelos autores em seus trabalhos e não na média de seus grupos. ${ }^{* * *}$ ) 86 é referente à soma de grupos amostrados, pelos diversos autores, 72 é relativo aos grupos descritos e que foram utilizados nas análises.

grupos de estudo. Porém, a visualização de indivíduos transeuntes de ambos os sexos (três fêmeas adultas e um macho subadulto - tabela I) corrobora outros estudos. Assim como em MeNDES (1989), a categoria FA engloba possíveis fêmeas sub-adultas, sendo que pode ser o caso das fêmeas transeuntes observadas neste estudo. Tal fato também foi observado por CALEGARO-MARQUES \& BICCA-MARQUES (1996) em A. caraya (Humboldt, 1812).

Em janeiro de 2004, ocorreu a morte/desaparecimento de um MA do Grupo Forninho provavelmente devido à caça ilegal. Fora esse desaparecimento, nenhum outro indivíduo desapareceu durante os estudos. Mesmo com o registro de duas predações de A. g. clamitans por jaguatirica (Leopardus pardalis (Linnaeus, 1758)), na área de estudo (dados não publicados). Além da jaguatirica, outros potenciais predadores estão presentes na área de estudo: irara (Eira barbara (Linnaeus, 1758)), suçuarana (Puma concoclor (Linnaeus, 1771)), gavião-pega-macaco (Spizaetus tyrannus (Wied-Neuwied, 1820)), gavião-pombo (Leucopternis polionota (Kaup, 1847)), águia-chilena (Geranoaetus melanoleucus (Vieillot, 1819)) e gavião-caboclo (Heterospizias meridionalis (Latham, 1790)). Destes, somente $S$. tyrannus foi observado em tentativas mal sucedidas de predação sobre os bugios (dados não publicados).

\section{Formação de grupos}

Embora seja um só registro, a fragmentação (ou fissão) do Grupo Pinheiro e a subseqüente formação de um novo grupo (Grupo Patropí), pode mostrar indícios de pelo menos uma das maneiras de como se dá a formação de novos grupos em $A$. g. clamitans. Outras maneiras de formação de grupos podem ser a partir do pareamento de indivíduos heterossexuais transeuntes ou a atração de indivíduos do sexo oposto por indivíduos solitários (CAlegaro-Marques \& Bicca-Marques 1996).

Embora as coletas de dados não tivessem este objetivo e, portanto, não obtiveram dados comprobatórios a este respeito, as observações oportunísticas sugerem que o macho "Patropí" separou-se de seu grupo original de uma forma gradativa e passou a ser visto nas áreas de vida de dois grupos (Grupo Pinheiro e Grupo Bica). Posteriormente foi seguido por uma FA, um JI e um MAS, os quais também se separam do grupo original de forma lenta e gradativa. Outra via de interpretação é que o MA (Patropí) tornou-se um indivíduo transeunte, por um curto período, atraindo outros indivíduos para formar seu próprio grupo. As duas interpretações deste caso, só diferem na teoria, pois na prática os eventos são os mesmos. A primeira hipótese (fissão de grupo) só pôde ser construída tendo em vista que os indivíduos agregados ao MA solitário, foram todos pertencentes ao grupo original do próprio MA.

Acredita-se que a fissão de grupos de A. g. clamitans possa ocorrer por um limite no número de indivíduos adultos, principalmente machos adultos, ligado à competição por acesso às fêmeas adultas. É possível observar grupos grandes que apresentam poucos indivíduos adultos e vários indivíduos jovens ou imaturos, como neste estudo no Grupo Forninho em 2004. Por outro lado, encontram-se também grupos grandes com muitos animais adultos, o que pode gerar certa instabilidade no grupo devido à competição, como no caso do Grupo 
Pinheiro que se desagregou formando dois novos grupos. Este também é o quadro observado por M. M. A. JARDim (dados não publicados) que correlaciona positivamente os desaparecimentos de indivíduos com o número de indivíduos por grupo. Esta pesquisadora também acredita numa instabilidade ligada ao número de indivíduos adultos por grupo.

\section{AGRADECIMENTOS}

Agradecemos ao Programa de Pós-Graduação em Zoologia, UFPR, pelo apoio no desenvolver este estudo. À CAPES pela bolsa de estudos concedida para este estudo (J.M.D.M.) e ao CNPq pela bolsa de pesquisador (F.C.P.). Ao Biólogo Édner L. Rosa, pela permissão de realizar esta pesquisa em sua propriedade; ao Biólogo Kauê C. Abreu e aos acadêmicos Itiberê P. Bernardi e Rodrigo F. Moro-Rios pelo auxílio em campo. Aos Pós-graduandos Lucas M. Aguiar e Gabriela Ludwig pelas correções e críticas dos originais.

\section{REFERÊNCIAS}

Aguiar, L.M.; N.R. Reis; G. Ludwig \& V.J. Rocha. 2003. Dieta, área de vida, vocalizações e estimativas populacionais de Alouatta guariba em um remanescente florestal no Norte do estado do Paraná. Neotropical Primates, Washington, 11 (2): 78-86.

Altmann, J. 1974. Observational study of behavior: sampling methods. Behaviour, Leiden, 40: 227-267.

Altmann, S.A. 1959. Field observations on a howling monkey society. Journal of Mammalogy, Lawrence, 40 (3): 317-330.

Auricchio, P. 1995. Primatas do Brasil. São Paulo, Terra Brasilis, $168 \mathrm{p}$.

BicCA-Marques, J.C. 2003. How do Howler Monkeys cope with habitat fragmentation?, p. 283-303. In: L.K. MARSH (Ed.). Primates in fragments: Ecology and Conservation. New York, Kluwer Academic, Plenum Publishers, 428p.

Bonvicino, C.R. 1989. Ecologia e comportamento de Alouatta belzebul (Primates: Cebidae) na Mata Atlântica. Revista Nordestina de Biologia, João Pessoa, 6 (2): 149-179.

BRocketT, R.C.; R.H. Horwich \& C.B. Jones. 2000. Female dispersal in the Belizean black howling monkey (Alouatta pigra). Neotropical Primates, Washington, 8 (1): 32-34.

Cabrera, A. 1957. Catalogo de los Mamiferos de America del Sur. Museo Argentino de Ciencias Naturales Bernardino Rivadavia e Instituto Nacional de Investigación de las Ciencias Naturales, Buenos Aires, 4 (1): 1-307.

Cabrera, A. \& J. Yepes. 1960. Mamiferos Sudamericanos. Buenos Aires, EDIAR, 238p.

Calegaro-Marques, C. \& J.C. Bicca-Marques. 1996. Emigration in a black howling monkey group. International Journal of Primatology, Chicago, 17 (2): 229-237.

Chiarello, A.G. 1993. Home range of the brown howler monkey, Alouatta fusca, in a forest fragment of Southeastern Brazil. Folia Primatologica, Basel, 60: 173-175.
Crockett, C. M. \& R. Rudran. 1987. Red howler monkey birth data. II. Interannual, habitat, and sex comparisons. American Journal of Primatology, Hoboken , 13: 369-384.

Dias, L.G. \& K.B. StRiER. 2003. Effects of group size on ranging patterns in Brachyteles arachnoides hypoxanthus. International Journal of Primatology, Chicago, 24 (2): 209-221.

Dunbar, R.I.M. 1988. Primate Social Systems. London, Croom Helm, 373p.

Emmons, L.H. \& F. Feer. 1997. Neotropical Rainforest Mammals. Chicago, University of Chicago Press, $2^{\text {nd }}$ ed., 307p.

ForTes, V.B. 2002. Um caso de rapto ou transferência acidental de um infante entre bandos vizinhos de Alouatta guariba clamitans. Neotropical Primates, Washington, 10 (3): 122-124.

Galetti, M., F. Pedroni \& M. Paschoal. 1994. Infanticide in the Brown howler monkey, Alouata fusca. Neotropical Primates, Washington, 2 (4): 6-7.

Jardim, M.M.A. \& L.F.B. Oliveira. 2000. Aspectos ecológicos e do comportamento de Alouatta fusca (Geoffroy, 1812) na Estação Ecológica de Aracurí, RS, Brasil, p. 151-169. In: C. Alonso \& A. Langguth (Eds). A Primatologia no Brasil. 7. João Pessoa, Editora Universitária UFPB, 360p.

Limeira, V.L.A. G. 2000. Uso do espaço por um grupo de Alouatta fusca clamitans em um fragmento degradado de Floresta Atlântica, p. 181-196. In: C. Alonso \& A. LangGuth (Eds). A Primatologia no Brasil. 7. João Pessoa, Editora Universitária, UFPB, 360p.

Marques, A.A.B. 2003. Primatas, p. 499-506. In: C. S. Fontana; G.A. BENCKE \& R.E. ReIs (Eds). Livro vermelho da fauna ameaçada de extinção no Rio Grande do Sul. Porto Alegre, EDIPUCRS, 632p.

Mendes, S.L.1989. Estudo ecológico de Alouatta fusca (Primates: Cebidae) na Estação Biológica de Caratinga, MG. Revista Nordestina de Biologia, João Pessoa, 6 (2): 71-104.

МiкICH, S.B. \& R.S. BÉRNILS. 2004. Livro vermelho da fauna ameaçada no Estado do Paraná. Governo do Paraná, SEMA/IAP, Curitiba, 763p.

Neville, M.K.; K.E. Glander; F. Braza \& A.B. Rylands. 1988. The howling monkeys, genus Alouatta, p. 349-453. In: R.A. Miтtermeier; A.B. Rylands; A. Coimbra-Filho \& G.A.B. Fonseca (Eds). Ecology and Behavior of Neotropical Primates. Washington, World Wildlife Fund, vol. 2, 610p.

NoWAK, R.M. 1999. Primates of the world. Baltimore, Johns Hopkins University Press, 224p.

Ostro, L.E.T.; S.C. Silver; F.W. Koontz; R.H. Horwich \& R. BROCKETT. 2001. Shifts in a social structure of black howler (Alouatta pigra) groups associated with natural and experimental variation in population density. International Journal of Primatology, Chicago, 22 (5): 733-748.

Rudran, R. \& E. Fernandez-Duque. 2003. Demographic changes over thirty years in a red howler population in Venezuela. International Journal of Primatology, Chicago, 24 (5): 925-947.

SiLva JR., E.C. 1981. A preliminary survey of brown howler monkeys (Alouatta fusca) at the Cantareira Reserve (São

Revista Brasileira de Zoologia 22 (1): 99-106, março 2005 
Paulo, Brazil). Revista Brasileira de Biologia, Rio de Janeiro, 41 (4): 897-909.

Silveira, R.M.M. \& T.L. CodenotTI. 2001. Interações sociais e dieta do bugio-ruivo, Alouatta guariba clamitans, no Parque Estadual de Itapuã, Rio Grande do Sul, Brasil. Neotropical Primates, Washington, 9 (1): 15-19.

Steinmetz, S. \& M. Souza. 1998. Unusual sexual postures in a howler monkey couple, Alouatta fusca clamitans. Neotropical Primates, Washington, 6 (2): 48-49.

Steinmetz, S. 2001. Densidade e conservação do Bugio (Alouatta fusca) no Parque Estadual Intervales. Neotropical Primates, Washington, 9 (2): 69-73.

Strier, K.B.; F.D.C. Mendes; J. Rímoli; A.O. Rímoli. 1993. Demogra-

Recebido em 08.VI.2004; aceito em 19.I.2005. phy and social structure of one group of muriquis (Brachyteles arachnoides). International Journal of Primatology, Chicago, 14 (4): 513-526.

Treves, A. 2001. Reproductive consequences of variation in the composition of howler monkey (Alouatta spp.) groups. Behavioral Ecology and Sociobiology, Heidelberg, 50 (1): 61-71.

von Ihering, H. 1914. Os bugios do gênero Alouatta. Revista do Museu Paulista, São Paulo, 9: 231-280.

WANG, E. \& K. Milton. 2003. Intragroup social relationships of male Alouatta palliata on Barro Colorado Island, Republic of Panama. International Journal of Primatology, Chicago, 24 (6): 1227-1243.

Revista Brasileira de Zoologia 22 (1): 99-106, março 2005 